\title{
Study on Colloidal Model of Petroleum Residues through the Attraction Potential between Colloids
}

\author{
Long-li Zhang, ${ }^{1,2}$ Guo-hua Yang, ${ }^{1}$ Chao-he Yang, ${ }^{2}$ and Guo-he Que ${ }^{2}$ \\ ${ }^{1}$ College of Science, China University of Petroleum (East China), Qingdao, Shandong 266555, China \\ ${ }^{2}$ State Key Laboratory of Heavy Oil Processing, China University of Petroleum (East China), Qingdao, Shandong 266555, China
}

Correspondence should be addressed to Long-li Zhang; 1lzhang@upc.edu.cn

Received 2 December 2015; Revised 2 March 2016; Accepted 3 March 2016

Academic Editor: Neil D. Danielson

Copyright (c) 2016 Long-li Zhang et al. This is an open access article distributed under the Creative Commons Attribution License, which permits unrestricted use, distribution, and reproduction in any medium, provided the original work is properly cited.

The samples of DaGang atmospheric residue (DG-AR), Middle East atmospheric residue (ME-AR), TaHe atmospheric residue (TH$\mathrm{AR}$ ), and their thermal reaction samples were chosen for study. All the samples were fractioned into six components separately, including saturates plus light aromatics, heavy aromatics, light resins, middle resins, heavy resins, and asphaltenes. The dielectric permittivity of the solutions of these components was measured, and the dielectric permittivity values of the components can be determined by extrapolation, which increased steadily from saturates plus light aromatics to asphaltenes. Moreover, the Hamaker constants of the components were calculated from their dielectric permittivity values. The Van der Waals attractive potential energy between colloids corresponding to various models could be calculated from the fractional composition and the Hamaker constants of every component. It was assumed that the cores of colloidal particles were formed by asphaltenes and heavy resins mainly; the other fractions acted as dispersion medium. For the three serials of thermal reaction samples, the Van der Waals attraction potential energy between colloids for this kind of model was calculated. For TH-AR thermal reaction samples, the Van der Waals attraction potential energy presented the maximum as thermal reaction is going on, which was near to the end of coke induction period.

\section{Introduction}

Petroleum residues are found to be colloidal systems, and asphaltenes are considered to be the cores of them [1-3]. Asphaltenes, which are the heaviest and the most polar fraction in residue, play a key role in the processing of heavy oil. The destruction of colloidal stability of heavy oil induces asphaltene precipitation and causes troubles to many petroleum processes including production, transportation, or refining.

Since the colloidal stability plays an important role in the usage of heavy oil, the colloidal model and colloidal stability of heavy oil have been widely studied. Because petroleum residue is a kind of complicated mixture, composed of innumerable molecules, it has to be divided into fractions based on polarity and solubility difference for further study [4-6]. Based on the solubility difference and chromatography separation, the former researchers found that the polarity of residue fractions increased steadily as the following sequence: saturates plus light aromatics, heavy aromatics, light resins, middle resins, heavy resins, and asphaltenes $[7,8]$. The fractions distribution of residue played an important role in the colloidal stability, the reaction characteristics, and the emulsification performance of residue [9].

Although many researchers have presented various micelle models for residue, there are no models based on the direct potential energy between colloids derived from the composition and characteristics of fractions. Moreover, the colloidal stability is influenced by the fractions distribution and the characteristics of heavy oil [10-13]. The mean dipole moment of the fractions can be measured [12]. For example, the researchers pointed out that SARA composition influenced the stability of asphaltenes in heavy oil [14-16]. But SARA composition partition is blurry, based on the solubility and polarity of molecules, and every fraction was a complicated mixture. Although the contents and the characteristic of SARA fractions determine the colloidal stability of heavy oil together, the relationship between them cannot be forecasted integrally and quantitatively. 
Some researchers pointed out that resins were helpful to the stability of asphaltenes $[15,16]$, so the increase of resin content would enhance the colloidal stability of heavy oil, and the increase of asphaltenes content would decrease the colloidal stability. It should be noted that resins are not required in order for asphaltenes to form stable nanocolloidal suspensions in toluene. As the division of asphaltene and resin is based on solubility, the characteristics of resin are adjacent to that of asphaltenes, and the characteristics of them are continuous. If the properties of the molecules are just on the critical point of asphaltenes and resins, it is difficult to deduce the effects of these molecules on the colloidal stability of residues, since this statement is qualitative and does not integrate the effect of properties and the composition of fractions of residue at the same time.

According to "the modified Yen model" proposed by Mullins and coresearchers [14], the asphaltene colloidal particles in crude oils were nanocolloidals, which were mainly composed of asphaltenes and the heaviest resins. The asphaltenes were in hierarchical structures in crude oils, such as molecules, nanoaggregates, and clusters of nanoaggregates. This work will study the attractive energy between the clusters dispersed in the medium of other fractions.

The colloidal stability of heavy oil is affected by the attractive potential and repulsive potential between colloid groups. This paper is to instruct the colloidal model of residue based on the attractive potentials between colloids for the sake of viewing the fractions distribution and fraction characteristics. This model will be used to reveal the colloidal stability variation during thermal reactions.

\section{Experimental Section}

There are three kinds of atmosphere residues studied, the boiling point of which was more than $350^{\circ} \mathrm{C}$. The samples were DaGang atmospheric residue (DG-AR), Middle East atmospheric residue (ME-AR), and TaHe atmospheric residue (TH-AR). Their properties have been presented in the previous papers $[8,12]$.

The samples were upgraded to study the colloidal stability variation of residues during thermal reaction. Thermal reaction was performed under nitrogen and the initial pressure is $1.0 \mathrm{MPa}$ at room temperature. The coke induction period of thermal reaction was $80 \mathrm{~min}$ for $\mathrm{DG}-\mathrm{AR}$ at $405^{\circ} \mathrm{C}, 100 \mathrm{~min}$ for ME-AR at $400^{\circ} \mathrm{C}$ [12], and $60 \mathrm{~min}$ for $\mathrm{TH}-\mathrm{AR}$ at $400^{\circ} \mathrm{C}$. The heavy oil was separated into n-pentane asphaltene and n-pentane maltene. Then, the maltene was separated into fractions through liquid chromatographic separation; and the neutron aluminum oxide was used as adsorbent, which was pretreated and contained 5\% wt. water [13]. The eluting solvents used were n-heptane, mixture of n-heptane and benzene $(\mathrm{v}: \mathrm{v}=85: 15)$, mixture of $\mathrm{n}$-heptane and benzene $(\mathrm{v}: \mathrm{v}=1: 1)$, benzene, or mixture of benzene and ethanol $(\mathrm{v}: \mathrm{v}=1: 1)$. The corresponding fractions could be gained as the following: saturates plus light aromatics, heavy aromatics, light resins, middle resins, and heavy resins. The colloidal stability variation of residue came from the shift of fractions distribution and the molecular characteristics of every fraction $[8,12]$.
The separation scheme, mean molecular weight, and the dielectric permittivity measuring methods were the same as described previously $[8,12,13]$.

The fractions were diluted by benzene separately. Firstly, a stock solution of fraction was prepared. Then, the stock solution was distributed in vials, and benzene was added to each vial to achieve the preconcerted weight percentage. The solution was placed at least for 24 hours before measurement to ensure stabilization. The dielectric permittivity of the solutions of fractions was measured, and the dielectric permittivity of fractions can be gained when extrapolated to the $100 \%$. The frequency used for permittivity measurements was $1 \mathrm{kHz}$. The Hamaker constants of the fractions were calculated from the dielectric characteristics of them. When the dielectric permittivity value is little, there are not notable differences between the long-range and the short-range Hamaker constant. The dielectric permittivity values of the components of petroleum residue were lower than 10 . So the short-range Hamaker constant was used in this study. When the two particles were the same and existed in vacuum, the short-range Hamaker constant can be calculated as [19]

$$
\begin{aligned}
A_{11} & =\frac{3 \sqrt{2}}{64 \pi} h \omega_{1} \frac{\left(\varepsilon_{1}-1\right)^{2}}{\left(\varepsilon_{1}+1\right)^{3 / 2}}=\frac{3 \sqrt{2}}{32} \frac{h c}{\lambda_{1}} \frac{\left(\varepsilon_{1}-1\right)^{2}}{\left(\varepsilon_{1}+1\right)^{3 / 2}} \\
& =A_{S M}
\end{aligned}
$$

$\varepsilon_{1}$ is the extrapolated relative dielectric permittivity of certain fraction; $\omega_{1}$ is the characteristic absorption frequency; $\lambda_{1}$ is the characteristic absorption wavelength, $\mathrm{nm}$; $h$ is the Planck constant, $6.626176 \times 10^{-34} \mathrm{~J} \cdot \mathrm{s} ; \mathrm{c}$ is the velocity of light, $3.0 \times$ $10^{8} \mathrm{~m} \cdot \mathrm{s}^{-1}$.

Since the fractions of petroleum residue were complex mixture, the UV-vis absorption spectrum of them was wideband from $200 \mathrm{~nm}$ to $400 \mathrm{~nm}$ without specific fine structures [20], the characteristic absorption wavelength for fractions of petroleum residue was chosen as $300 \mathrm{~nm}$. The Hamaker constants of fractions could be computed by (1). The researchers have proposed various colloidal model of residue $[14,17,18]$. The Van der Waals attraction potential energy of different model could be calculated from the fractional composition and the Hamaker constants of every component.

\section{Results and Discussions}

3.1. The Dielectric Permittivity of the Solutions of Fractions. The dielectric permittivity of the solutions of fractions was measured. The asphaltene of TH-AR thermal reaction sample for 40 minutes was illustrated, as exampled. It could be seen from Figure 1 that the dielectric permittivity of solution increased with concentration, and the value for the neat material can be determined by extrapolation to $100 \%$, which was 7.75. The dielectric permittivity of other samples could be gained also and shown in Tables 1-3.

It can be seen in Tables 1-3 that the dielectric permittivity values increased following the sequence, saturates plus light aromatics, heavy aromatics, light resins, middle resins, heavy resins, and asphaltenes, which was consistent with the polarity variation of them. Furthermore, all dielectric permittivity 
TABLE 1: Dielectric permittivity of samples of DG-AR thermal reaction at $405^{\circ} \mathrm{C}$.

\begin{tabular}{lcccccc}
\hline Reaction time/min & $\begin{array}{c}\text { Saturates plus } \\
\text { light aromatics }\end{array}$ & Heavy aromatics & Light resins & Middle resins & Heavy resins & Asphaltenes \\
\hline DG-AR & 2.52 & 3.49 & 3.48 & 4.79 & 5.76 & 7.38 \\
40 & 2.29 & 3.04 & 3.41 & 4.01 & 5.38 & 6.65 \\
80 & 2.40 & 3.07 & 3.18 & 3.61 & 5.19 & 7.99 \\
120 & 2.40 & 3.10 & 3.27 & 4.16 & 5.23 & 10.20 \\
160 & 2.44 & 2.80 & 3.56 & 4.00 & 5.30 & 9.13 \\
\hline
\end{tabular}

TABLE 2: Dielectric permittivity of samples of ME-AR thermal reaction at $400^{\circ} \mathrm{C}$.

\begin{tabular}{lcccccc}
\hline Reaction time/min & $\begin{array}{c}\text { Saturates plus } \\
\text { light aromatics }\end{array}$ & Heavy aromatics & Light resins & Middle resins & Heavy resins & Asphaltenes \\
\hline ME-AR & 2.50 & 2.94 & 3.79 & 4.68 & 5.30 & 9.21 \\
40 & 2.58 & 3.43 & 3.77 & 3.57 & 4.84 & 8.83 \\
80 & 2.46 & 3.14 & 4.11 & 4.89 & 5.17 & 5.86 \\
100 & 2.50 & 4.76 & 4.68 & 5.65 & 5.49 & 7.39 \\
160 & 2.47 & 3.09 & 3.97 & 5.54 & 7.56 \\
\hline
\end{tabular}

TABLE 3: Dielectric permittivity of samples of TH-AR thermal reaction at $400^{\circ} \mathrm{C}$.

\begin{tabular}{|c|c|c|c|c|c|c|}
\hline Reaction time/min & $\begin{array}{l}\text { Saturates plus } \\
\text { light aromatics }\end{array}$ & Heavy aromatics & Light resins & Middle resins & Heavy resins & Asphaltenes \\
\hline TH-AR & 2.46 & 2.85 & 3.24 & 4.30 & 5.51 & 6.79 \\
\hline 40 & 2.50 & 3.06 & 3.91 & 4.36 & 6.22 & 7.75 \\
\hline 80 & 2.39 & 3.58 & 4.19 & 4.30 & 5.08 & 8.53 \\
\hline 120 & 2.54 & 3.85 & 4.13 & 5.55 & 4.99 & 6.32 \\
\hline 160 & 2.34 & 3.35 & 4.06 & 5.13 & 5.51 & 5.37 \\
\hline
\end{tabular}

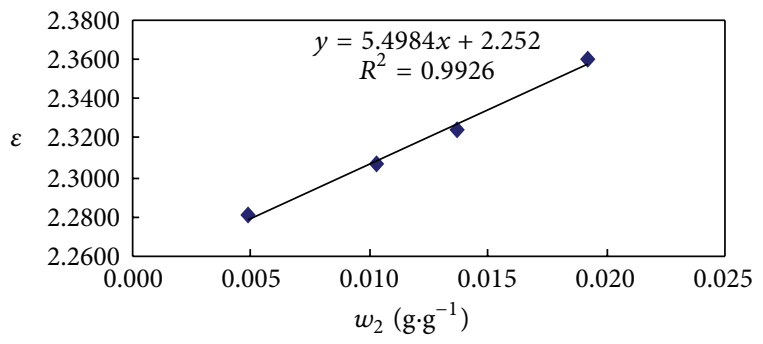

FIGURE 1: The dielectric permittivity values as a function of concentration for solution of asphaltenes of TH-AR thermal reaction sample for $40 \mathrm{~min}$.

values were lower than 10 , much lower compared to water which was 78. From the low dielectric permittivity values determined, the Hamaker constants of fractions could be calculated by (1) [19].

3.2. The Van Der Waals Attractive Potential Energy between Colloids for Different Model. The Van der Waals attractive potential energy between colloids for different models can be calculated by Hamaker constant of the fractions and the fractions distribution. The colloidal model with the lowest energy will be the most stable construction in view of attractive energy. Seven kinds of models would be put forward for comparison.

Vold deduced the effect of solvation sheaths on the Van der Waals attraction between spherical colloid particles [21], which can be calculated as (2)-(5) and were shown in Figure 2 [21-23]:

$$
\begin{aligned}
-12 V= & \sum_{i, j=1, n} a_{i} a_{j} H_{i j}, \\
a_{i}= & \left(A_{i}^{1 / 2}-A_{i-1}{ }^{1 / 2}\right)^{2}, \\
H_{i j}= & H_{j i}=H\left(\frac{\delta_{i j}}{2 R_{k}}, \frac{R_{l}}{R_{k}}\right), \\
H(x, y)= & \frac{y}{x^{2}+x y+x}+\frac{y}{x^{2}+x y+x+y} \\
& +2 \ln \left(\frac{x^{2}+x y+x}{x^{2}+x y+x+y}\right) .
\end{aligned}
$$

The subscript zero was assigned to the medium and successive layers of adsorbed material are numbered from the outside inward as $1,2, \ldots, n-1$; the unsolvated particle is numbered $n$. 


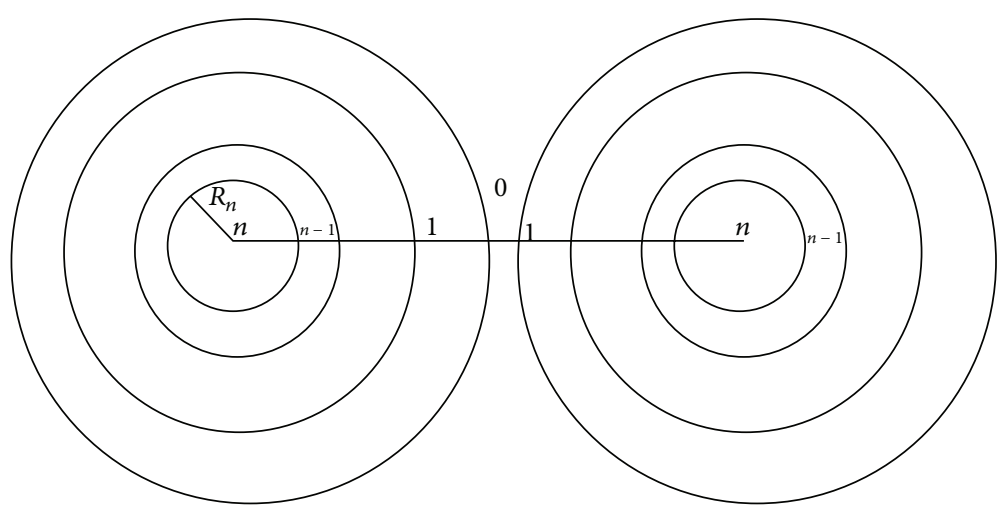

FIgURE 2: The diagram of two particles with adsorbed layers.

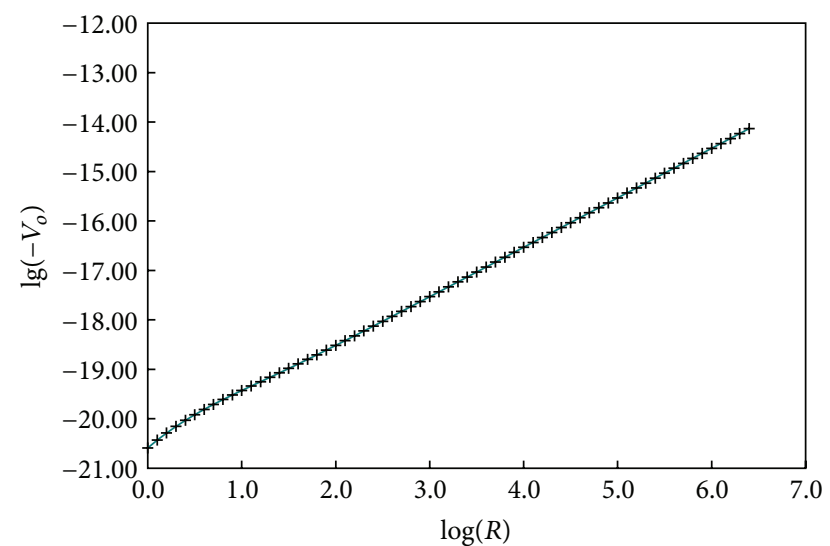

FIGURE 3: The variation of attractive potential energy with radius values of TH-AR for model \#7.

$A_{i}$ is the Hamaker constant of the shell numbered $i$.

$R_{k}$ is the radius of the shell $i$ or $j$, which has the smaller radius, $R_{l}$ is the other, and $\delta_{i j}$ is the separation of the surface of the two spheres. $H$ is the functor defined by (4) and (5).

The seven kinds of models were presented in Table 4. The asphaltenes, which were the largest and most aromatic molecules, were in hierarchical nanocolloidal structures in heavy oils.

For the model \#2, when the radius of asphaltene core was considered $10 \mathrm{~nm}$, the heavy resin adsorbed layer thickness can be computed based on the relative content of them. Then, the thickness of other adsorbed layers could be computed. The distance between the outer surfaces is fixed as $0.3 \mathrm{~nm}$ [22]. The attractive energy potential can be computed from the Hamaker constants and the composition of fractions for certain sample, at certain model, and for certain radius of cores.

As the radius of asphaltene cores varied from $1.0 \mathrm{~nm}$ to larger values, the attractive potential energy at different radius values for TH-AR of model \#7 could be calculated, which was shown in Figure 3.

It can be seen in Figure 3 that the attractive potential energy increased with the increase of radius of asphaltene cores. To compare the attractive potential energy of different

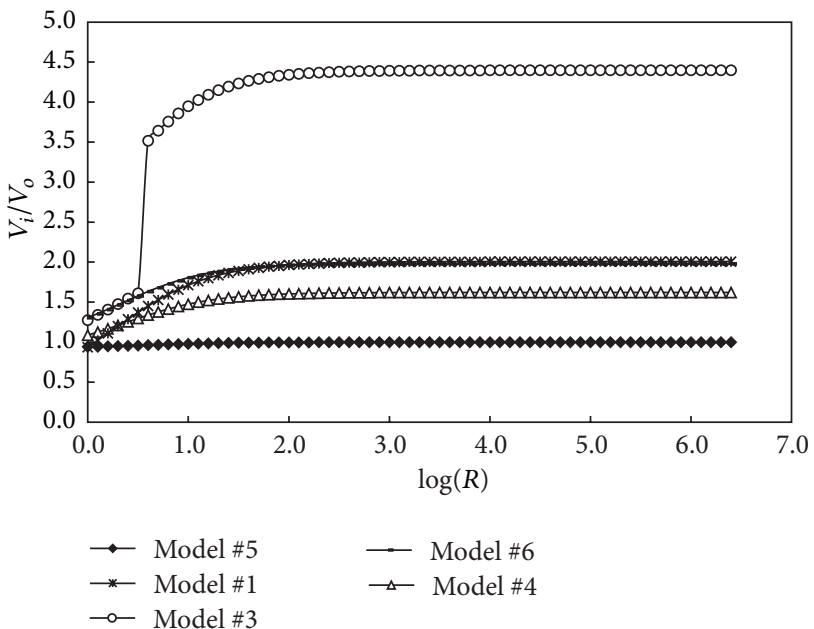

FIGURE 4: The ratios of the attractive potential energy for different model to that of model \#7 for DG-AR.

model, the ratios of the attractive potential energy of different model to that of model \#7 were calculated at different colloidal radius and shown in Figure 4.

It can be seen in Figure 4 that, for DG-AR, the energy for model \#5 approximately equals to 1 , and the others were more than 1 . So model \#5 and model \#7 were the most possible models for DG-AR in view of attractive potential energy. Most of the other studied samples presented the same phenomenon.

Furthermore, all the samples were studied and the result showed that the model $\# 2$ was the most stable model for almost all the samples, except for two thermal reaction products from ME-AR.

The modified Yen model is confirmed and widely accepted [14]; the attractive potential energy under model \#7 was calculated and shown in Tables 5-7, assuming that the asphaltene radius equaling $10 \mathrm{~nm}$ or $50 \mathrm{~nm}$. The colloidal stability parameters of the residual samples were characterized through mass fraction normalized conductivity method [12]. The coke induction period of thermal reaction was $80 \mathrm{~min}$ for DG-AR, $100 \mathrm{~min}$ for ME-AR, and $60 \mathrm{~min}$ for TH-AR. 
TABLE 4: The suggested colloidal model.

\begin{tabular}{|c|c|c|c|c|}
\hline & Cores & Absorbent & Medium & Proposed by \\
\hline Model \#1 & Asphaltenes & & $\begin{array}{c}\text { Heavy resins, middle resins, light resins, } \\
\text { heavy aromatics, and saturates plus light } \\
\text { aromatics }\end{array}$ & Mack [17] \\
\hline Mode \#2 & Asphaltenes & $\begin{array}{l}\text { Heavy resins, middle } \\
\text { resins, light resins, } \\
\text { and heavy aromatics, } \\
\text { steadily }\end{array}$ & Saturates plus light aromatics & Pfeiffer and Van Doormaal [18] \\
\hline Mode \#3 & $\begin{array}{l}\text { Asphaltenes and } \\
\text { heavy resins }\end{array}$ & & $\begin{array}{c}\text { Heavy resins, middle resins, light resins, } \\
\text { heavy aromatics, and saturates plus light } \\
\text { aromatics }\end{array}$ & \\
\hline Mode \#4 & Asphaltenes & Heavy resins & $\begin{array}{c}\text { Middle resins, light resins, heavy } \\
\text { aromatics, and saturates plus light } \\
\text { aromatics }\end{array}$ & \\
\hline Mode \#5 & Asphaltenes & $\begin{array}{l}\text { Heavy resins and } \\
\text { middle resins }\end{array}$ & $\begin{array}{l}\text { Light resins, heavy aromatics, and } \\
\text { saturates plus light aromatics }\end{array}$ & \\
\hline Mode \#6 & $\begin{array}{l}\text { Asphaltenes, heavy } \\
\text { resins, and middle } \\
\text { resins }\end{array}$ & & $\begin{array}{l}\text { Light resins, heavy aromatics, and } \\
\text { saturates plus light aromatics }\end{array}$ & \\
\hline Mode \#7 & $\begin{array}{l}\text { Asphaltenes and } \\
\text { heavy resins }\end{array}$ & Middle resins & $\begin{array}{l}\text { Light resins, heavy aromatics, and } \\
\text { saturates plus light aromatics }\end{array}$ & Mullins [14] \\
\hline
\end{tabular}

TABLE 5: The attractive energy between colloids of TH-AR for model \#7.

\begin{tabular}{lccc}
\hline Reaction time/min & $\begin{array}{c}\text { The energy when } \\
R=10 \mathrm{~nm}(\mathrm{~J})\end{array}$ & $\begin{array}{c}\text { The energy when } \\
R=50 \mathrm{~nm}(\mathrm{~J})\end{array}$ & Colloidal stability parameters \\
\hline 0 (TH-AR) & $-3.74 \times 10^{-20}$ & $-1.58 \times 10^{-19}$ & 1.45 \\
40 & $-4.45 \times 10^{-20}$ & $-1.60 \times 10^{-19}$ & 0.82 \\
80 & $-5.01 \times 10^{-20}$ & $-1.72 \times 10^{-19}$ & 0.38 \\
120 & $-4.79 \times 10^{-20}$ & $-2.59 \times 10^{-19}$ & 0.28 \\
160 & $-4.87 \times 10^{-20}$ & $-2.72 \times 10^{-19}$ & 0.20 \\
\hline
\end{tabular}

TABLE 6: The attractive energy between colloids of DG-AR for model \#7.

\begin{tabular}{lccc}
\hline Reaction time/min & $\begin{array}{c}\text { The energy when } \\
R=10 \mathrm{~nm}(\mathrm{~J})\end{array}$ & $\begin{array}{c}\text { The energy when } \\
R=50 \mathrm{~nm}(\mathrm{~J})\end{array}$ & Colloidal stability parameters \\
\hline DG-AR & $-5.45 \times 10^{-20}$ & $-2.86 \times 10^{-19}$ & 8.20 \\
40 & $-3.65 \times 10^{-20}$ & $-1.70 \times 10^{-19}$ & 2.29 \\
80 & $-2.65 \times 10^{-20}$ & $-9.68 \times 10^{-20}$ & 1.14 \\
120 & $-4.48 \times 10^{-20}$ & $-1.78 \times 10^{-19}$ & 0.67 \\
160 & $-3.65 \times 10^{-21}$ & $-1.44 \times 10^{-19}$ & 0.71 \\
\hline
\end{tabular}

TABLE 7: The attractive energy between colloids of ME-AR for model \#7.

\begin{tabular}{lccc}
\hline Reaction time/min & $\begin{array}{c}\text { The energy when } \\
R=10 \mathrm{~nm}(\mathrm{~J})\end{array}$ & $\begin{array}{c}\text { The energy when } \\
R=50 \mathrm{~nm}(\mathrm{~J})\end{array}$ & Colloidal stability parameters \\
\hline ME-AR & $-5.18 \times 10^{-20}$ & $-2.49 \times 10^{-19}$ & 1.70 \\
40 & $-2.05 \times 10^{-20}$ & $-6.31 \times 10^{-20}$ & 1.22 \\
80 & $-4.80 \times 10^{-20}$ & $-2.42 \times 10^{-19}$ & 1.04 \\
100 & $-6.07 \times 10^{-20}$ & $-3.19 \times 10^{-19}$ & 0.86 \\
160 & $-6.76 \times 10^{-20}$ & $-3.52 \times 10^{-19}$ & 0.60 \\
\hline
\end{tabular}


TABLE 8: The electric repulsive potential energy between colloids.

\begin{tabular}{lcccc}
\hline Sample & $\xi(\mathrm{mV})$ & $R(\mathrm{~nm})$ & $U_{R}(\mathrm{~J})$ & Attractive potential energy $(R=50 \mathrm{~nm})$ \\
\hline $0($ ME-AR $)$ & -41.77 & $55 \pm 1.25$ & $1.06 \times 10^{-21}$ & $-2.49 \times 10^{-19}$ \\
ME-AR, 40 min & 26.91 & $145 \pm 1.25$ & $1.16 \times 10^{-21}$ & $-6.31 \times 10^{-20}$ \\
ME-AR, 100 min & 31.79 & $180 \pm 1.25$ & $2.01 \times 10^{-21}$ & $-3.19 \times 10^{-19}$ \\
\hline
\end{tabular}

It can be seen from Tables 5-7 that for the studied samples, as thermal reaction is going on, the colloidal stability parameters decreased steadily. It can be seen from Table 5 that for the TH-AR thermal reaction samples, when radii are equaling $50 \mathrm{~nm}$, the attractive potential energy between colloids increased as thermal reaction is going on, which was consistent with the colloidal stability parameters decreasing. For the TH-AR thermal reaction samples, when radii are equaling $10 \mathrm{~nm}$, the attractive potential energy got to the maximum at 80 minutes, which was almost the same to the end of coke induction period and decreased after that, as thermal reaction is going on. The coke induction period was defined to be the time at which $0.1 \mathrm{wt} \%$ coke was produced.

It can be seen from Table 6 that, for the DG-AR thermal reaction samples, the attractive potential energy presented the maximum at 120 minutes, except for the DG-AR, no matter whether radii are equaling $10 \mathrm{~nm}$ or $50 \mathrm{~nm}$. It can be seen from Table 7 that, for the ME-AR thermal reaction samples, the attractive potential energy presented an increasing tendency except for the ME-AR, no matter whether radii are equaling $10 \mathrm{~nm}$ or $50 \mathrm{~nm}$. The possible explanation is that the colloidal stability is determined by the attractive energy and the repulsive energy.

Finally, the repulsive potential energy was studied. The Zeta potentials of the solutions of ME-AR and thermal reaction samples were measured by a Phase Analysis Light Scattering Potential Analyzer. The electric repulsive potential energy between residue colloids was computed and shown in Table 8 [24].

It can be seen in Table 8 that the dielectric repulsive potential energy between residue colloids was far less than that of attractive potential energy, which was about one tenth of the latter. The attractive potential energy values were determined supposing that the radii were $50 \mathrm{~nm}$, and the expected attractive potential energy values would be bigger as the radii were larger than $50 \mathrm{~nm}$. Perhaps the stabilization effect mainly came from the steric repulsive strength from alkyl chain of the asphaltenes and resins. So the key factors for colloidal stability of residue are Van der Waals attractive potential energy and steric repulsive potential energy.

\section{Conclusion}

Firstly, the results showed that the modified Yen model proposed by Mullins provided lower attractive potential energy for most residues.

Secondly, for TH-AR thermal reaction samples when radii of cores are equaling $50 \mathrm{~nm}$ and for $\mathrm{ME}-\mathrm{AR}$ thermal reaction thermal samples except for ME-AR, the Van der Waals attractive potential energy increased steadily as thermal reaction is going on, which was consistent with the colloidal stability parameter decreasing. These phenomena showed that the attractive potential energy played a key role in the colloidal stability of residue.

Thirdly, the dielectric repulsive potential energy was far less than the Van der Waals attractive potential energy between residual colloids, indicating that the key factors for colloidal stability of residue are Van der Waals attractive potential energy and steric repulsive potential energy.

\section{Competing Interests}

The authors declare that they have no competing interests.

\section{Acknowledgments}

This work was partially supported by the National Science Fund Committee of China (20776160, 21576292) and the Fundamental Research Funds for the Central Universities (14CX05029A).

\section{References}

[1] J. P. Dickie and T. F. Yen, "Macrostructures of the asphaltic fractions by various instrumental methods," Analytical Chemistry, vol. 39, no. 14, pp. 1847-1852, 1967.

[2] T. F. Yen, "The colloidal aspect of a macrostructure of petroleum asphalt," Fuel Science and Technology International, vol. 10, no. 4-6, pp. 723-733, 1992.

[3] E. Y. Sheu, "Petroleum asphaltene-properties, characterization, and issues," Energy \& Fuels, vol. 16, no. 1, pp. 74-82, 2002.

[4] I. A. Wiehe, "A phase-separation kinetic model for coke formation," Industrial and Engineering Chemistry Research, vol. 32, no. 11, pp. 2447-2454, 1993.

[5] D. A. Storm and E. Y. Sheu, "Characterization of colloidal asphaltenic particles in heavy oil," Fuel, vol. 74, no. 8, pp. 1140$1145,1995$.

[6] S. Li, C. Liu, G. Que, W. Liang, and Z. Yajie, "Colloidal structures of three Chinese petroleum vacuum residues," Fuel, vol. 75, no. 8, pp. 1025-1029, 1996.

[7] P. Wattana, H. S. Fogler, A. Yen, M. Del Carmen Garcia, and L. Carbognani, "Characterization of polarity-based asphaltene subfractions," Energy \& Fuels, vol. 19, no. 1, pp. 101-110, 2005.

[8] L. L. Zhang, G. H. Yang, G. H. Que, C. H. Yang, and H. H. Shan, "Dipole moment variation of a petroleum residue during catalytic and thermal upgrading," Energy \& Fuels, vol. 23, no. 4, pp. 2086-2089, 2009.

[9] O. C. Mullins, E. Y. Sheu, A. Hammami, and A. G. Marshall, Asphaltenes, Heavy Oil, and Petroleomics, Schlumberger, New York, NY, USA, 2007.

[10] O. León, E. Rogel, J. Espidel, and G. Torres, "Asphaltenes: structural characterization, self-association, and stability behavior," Energy and Fuels, vol. 14, no. 1, pp. 6-10, 2000. 
[11] N. F. Carnahan, J.-L. Salager, R. Antón, and A. Dávila, "Properties of resins extracted from Boscan crude oil and their effect on the stability of asphaltenes in Boscan and Hamaca crude oils," Energy and Fuels, vol. 13, no. 2, pp. 309-314, 1999.

[12] L. L. Zhang, G. H. Yang, G. H. Que, Q. X. Zhang, and P. J. Yang, "Colloidal stability variation of petroleum residue during thermal reaction," Energy \& Fuels, vol. 20, no. 5, pp. 2008-2012, 2006.

[13] C. G. Liu, G. H. Que, Y. Z. Chen, and W. J. Liang, "Evaluating VR using liquid chromatogram and ${ }^{1} \mathrm{H}-\mathrm{NMR}$ spectrum," Acta Petrolei Sinica (Petroleum Processing Section), vol. 3, pp. 90-98, 1987.

[14] O. C. Mullins, “The modified yen model," Energy and Fuels, vol. 24, no. 4, pp. 2179-2207, 2010.

[15] P. M. Spiecker, K. L. Gawrys, C. B. Trail, and P. K. Kilpatrick, "Effects of petroleum resins on asphaltene aggregation and water-in-oil emulsion formation," Colloids and Surfaces A: Physicochemical and Engineering Aspects, vol. 220, no. 1-3, pp. 9-27, 2003.

[16] H. W. Yarranton, W. A. Fox, and W. Y. Svrcek, "Effect of resins on asphaltene self-association and solubility," Canadian Journal of Chemical Engineering, vol. 85, no. 5, pp. 635-642, 2007.

[17] C. Mack, "Colloidal chemistry of asphalts," Journal of Physics Chemistry, vol. 36, pp. 2901-2914, 1932.

[18] J. P. Pfeiffer and P. M. Van Doormaal, “The rheological properties of asphaltic bitumen," Journal of the Institution of Petroleum Technologists, vol. 22, no. 152, pp. 414-440, 1936.

[19] Z. Zheng and N. Li, The Molecular Strength and the Stability or Aggregation of Colloids, Higher Education Press, Beijing, China, 1995.

[20] W. J. Liang, The Chemistry of Heavy Oil, Press of University of Petroleum, Dongying, China, 2000.

[21] M. J. Vold, "The effect of adsorption on the van der waals interaction of spherical colloidal particles," Journal of Colloid Science, vol. 16, no. 1, pp. 1-12, 1961.

[22] D. W. J. Osmond, B. Vincent, and F. A. Waite, “The van der Waals attraction between colloid particles having adsorbed layers. I. A reappraisal of the 'Vold effect"', Journal of Colloid And Interface Science, vol. 42, no. 2, pp. 262-269, 1973.

[23] B. Vincent, "The Van der Waals attraction between colloid particles having adsorbed layers. II. Calculation of interaction curves," Journal of Colloid and Interface Science, vol. 42, no. 2, pp. 270-285, 1973.

[24] L. L. Zhang, G. H. Yang, and G. H. Que, "Study on the Zeta potential of Middle East atmospheric residue and thermal reaction samples," Journal of Fuel Chemistry and Technology, vol. 33, no. 1, pp. 125-128, 2005. 

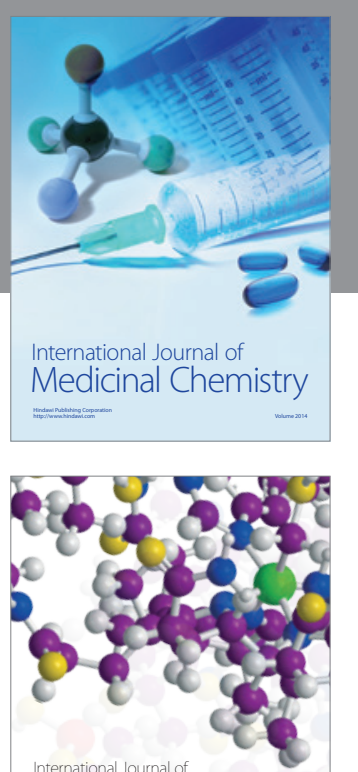

Carbohydrate Chemistry

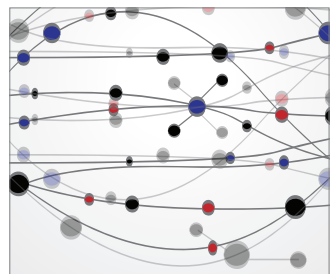

The Scientific World Journal
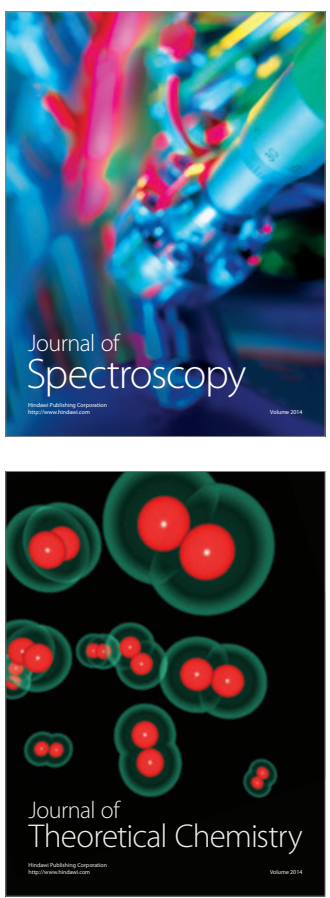
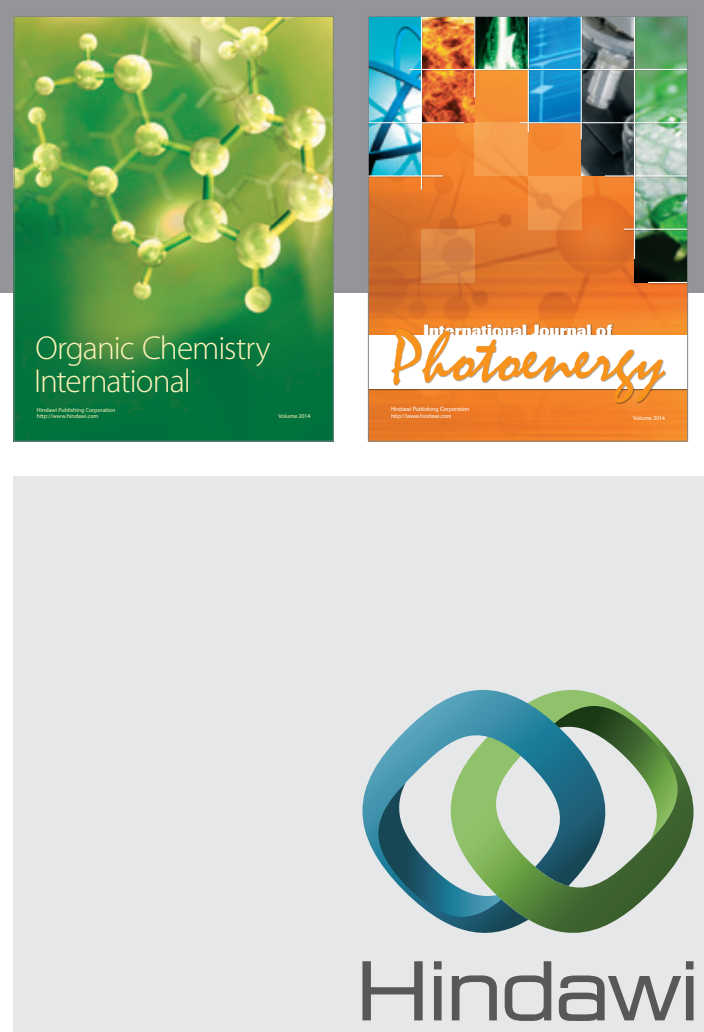

Submit your manuscripts at

http://www.hindawi.com

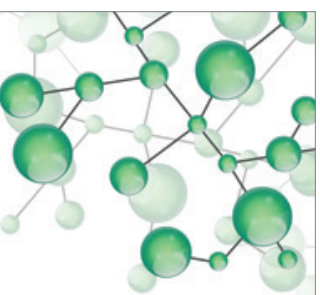

International Journal of

Inorganic Chemistry

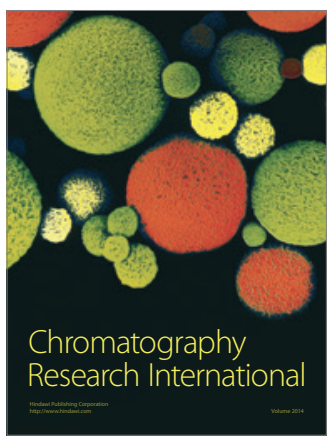

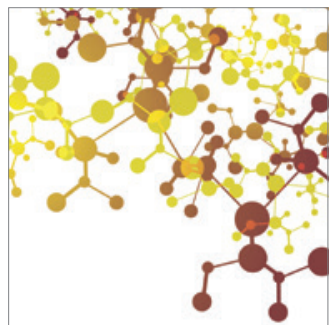

Applied Chemistry
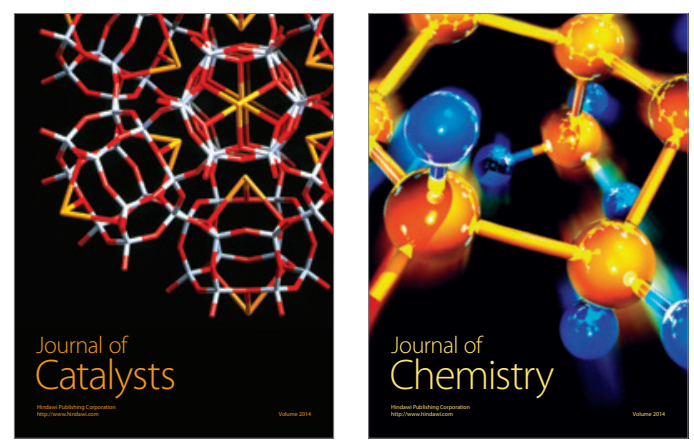
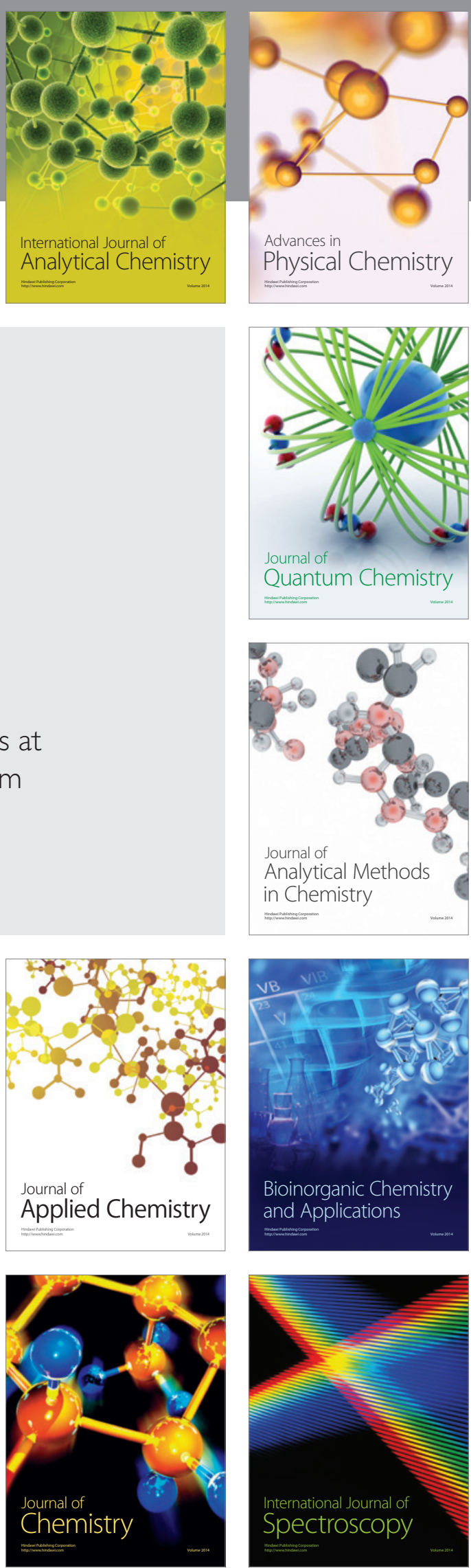